\title{
Using the hybrid EMD-BPNN model to predict the incidence of HIV in Dalian, Liaoning Province, China, 2004-2018
}

Qingyu $\mathrm{An}^{{ }^{*}}{ }^{\mathbb{C}}$, Jun $\mathrm{Wu}^{1}$, Jun Meng ${ }^{1}$, Zhijie Zhao ${ }^{1}$, Jin Jian Bai ${ }^{1}$ and Xiaofeng $\mathrm{Li}^{2}$

\begin{abstract}
Background: Acquired immunodeficiency syndrome (AIDS) is a malignant infectious disease with high mortality caused by HIV (human immunodeficiency virus, and up to now there are no curable drugs or effective vaccines. In order to understand AIDS's development trend, we establish hybrid EMD-BPNN (empirical modal decomposition and Back-propagation artificial neural network model) model to forecast new HIV infection in Dalian and to evaluate model's performance.
\end{abstract}

Methods: The monthly HIV data series are decomposed by EMD method, and then all decomposition results are used as training and testing data to establish BPNN model, namely BPNN was fitted to each IMF (intrinsic mode function) and residue separately, and the predicted value is the sum of the predicted values from the models. Meanwhile, using yearly HIV data to established ARIMA and using monthly HIV data to established BPNN, and SARIMA (seasonal autoregressive integrated moving average) model to compare the predictive ability with EMD-BPNN model.

Results: From 2004 to 2017, 3310 cases of HIV were reported in Dalian, including 101 fatal cases. The monthly HIV data series are decomposed into four relatively stable IMFs and one residue item by EMD, and the residue item showed that the incidence of HIV increases firstly after declining. The mean absolute percentage error value for the EMD-BPNN, BPNN, SARIMA $(1,1,2)(0,1,1)$ in 2018 is $7.80 \%, 10.79 \%, 9.48 \%$ respectively, and the mean absolute percentage error value for the ARIMA $(3,1,0)$ model in 2017 and 2018 is $8.91 \%$.

Conclusions: The EMD-BPNN model was effective and reliable in predicting the incidence of HIV for annual incidence, and the results could furnish a scientific reference for policy makers and health agencies in Dalian.

Keywords: HIV, EMD, BPNN, ARIMA

\section{Background}

The full name of AIDS is acquired immunodeficiency syndrome, which is a malignant infectious disease with high mortality caused by human immunodeficiency virus infection [1], and has become one of global public health problem [2]. Since the first case reported in China [3], there has been a tendency toward an increase in HIV

\footnotetext{
*Correspondence: anqingyu@163.com

${ }^{1}$ Dalian Center for Disease Control and Prevention, Dalian 116021,

Liaoning, China

Full list of author information is available at the end of the article
}

epidemics in the country [4]. As of September 30 in 2013, a total of 434,000 HIV infections and AIDS patients were reported in China, and the main way of transmission in China was transsexual transmission at present [5]. Up to now, there are no curable drugs or effective vaccines. Therefore, establish forecasting model of HIV, so as to find the development trend of HIV, is of great significance to the HIV prevention and control work. For the epidemic trend of HIV, its influencing factors are complex, including population, economy, behavior and environment. At present, China has not fully carried out the 
monitoring and collection of data on HIV-related influencing factors, so it is difficult to establish a prediction model of HIV by analyzing the influencing factors. In recent years, some scholars have used ARIMA model, GM $(1,1)$ model, BP neural network model to predict the incidence trend of HIV. For example, Liang et al. [6] using $G(1,1)$ modeling method to fit the incidence of HIV in Jiangsu province, the relative error was $23.89 \%$. GM is a mathematical model based on grey system theory, which can systematically predict the trend of variable change. GM $(1,1)$ is the simplest form of model, the basic steps of establishing the model are firstly cumulate the irregular original data to a regular sequence of data and then build the differential equation, so as to predict the future development trend of the disease [7]. Yang et al. [8] using ARIMA to build modeling HIV incidence from 2000 to 2014 in China and the mean absolute percentage error was $19.90 \%$. Wu et al. [9] use Back-propagation neural network (BP-ANN) as a model to predict HIV prevalence, and the ratios of accuracy for training, calibration and detection were $93.94 \%, 88.48 \%$ and $89.60 \%$, respectively.

In order to improve the accuracy of the prediction model, in this study, we took the HIV incidence data from 2004 to 2018 as an example, established a two-stage EMD (empirical mode decomposition)-BPNN (back-propagation artificial neural network model) model to forecast HIV in Dalian and the prediction results will provide the basis for AIDS monitoring and prevention in Dalian.

\section{Materials and methods}

\section{Study area}

Dalian is the main coastal city of Liaoning Province, China and a major tourist city located at $38^{\circ} 43^{\prime}-40^{\circ}$ $10^{\prime} \mathrm{N}$ latitude and $120^{\circ} 58^{\prime}-123^{\circ} 31^{\prime} \mathrm{E}$ longitude. It had a registered population of 5.949 million in 2017. Dalian has a warm continental monsoon climate and is in a marine temperate zone. The average annual temperature is $10{ }^{\circ} \mathrm{C}$ with a maximum of $35{ }^{\circ} \mathrm{C}$, and a minimum of $-28 \sim-18{ }^{\circ} \mathrm{C}$. The average rainfall is $550-800 \mathrm{~mm}$ and the total hours of annual sunshine is $2500-2800 \mathrm{~h}$ [10].

\section{Data collection}

The HIV was a notifiable monitoring communicable disease in China. The clinicians are required to report HIV cases through the China information system for disease control and prevention within $24 \mathrm{~h}$. During 1995 to 2008, there was 320 cases of HIV infection reported in Dalian, and the average annual growth rate of incidence was $31.06 \%$ [11]. Yearly incidence rate of HIV during the period of 1999 to 2018 and monthly incidence number of HIV cases in Dalian during the period of 2004 to 2018 was obtained from above information system's statistical report around the beginning of February in the next year. The diagnostic criteria of HIV is compliance with diagnostic criteria of HIV infection by Secretariat of the Professional Committee on Infectious Diseases Standards and Secretariat of the Professional Committee on Parasitic Disease Standards of the Ministry of Health of the People's Republic of China in May, 2010 [12].

\section{Data analysis}

The monthly HIV data series are decomposed by EMD method, and then all decomposition results are used as training and testing data to establish BPNN model, namely BPNN was fitted to each intrinsic mode function (IMF) and residue separately, the predicted value is the sum of the predicted values from the models, and the annual incidence equal to the sum of the predicted results of the 12 months. Meanwhile, using monthly HIV data to established BPNN and SARIMA model and using yearly HIV incidence rate to established ARIMA model to compare the predictive ability with EMD-BPNN model.

\section{Empirical modal decomposition}

Empirical modal decomposition is a self-adaptive decomposition method which is developed for non-stationary and nonlinear signal processing, was proposed by Huang et al. [13]. After EMD processing, complex signals can be decomposed into several intrinsic mode function (IMF) components based on the local characteristic time scale of the signal from high to low frequency and a residue.

The expression of the model is:

$$
s(t)=\sum_{t=1}^{n} i m f i(t)+r n(t)
$$

where $s(t)$ denote original signals, imfi $(t)$ denote the $i$ th intrinsic mode function, and $i=1,2, \cdots, n, r n(t)$ denote residual signal.

The IMF must meet the following two conditions:

1. In the whole signal data series, the number of extrema points must be equal to the number of Zero or differ by one at most;

2. The mean value of envelope defined by maximum and minimum must be equal and zero [14].

Empirical modal decomposition of monthly incidence data of HIV from 2004 to 2017 was performed by using MATLAB 2014.

\section{BP artificial neural network}

A back-propagation artificial neural network is a multilayer feed-forward neural network trained by error back 
propagation algorithm [15]. Its topological structure consists of three layers, namely input layer, hidden layer and output layer, each of which consists of some joints. The hidden layer connects input and output layer, and their correlations are reflected by relevant coefficients, namely connection weights. Usually, through self-learning or self-training of artificial neural network, repeatedly adjust these coefficients until reaching a goal of welltrained model (Fig. 1).

The learning process of BP algorithm consists of two processes: forward propagation and backward propagation. In the forward propagation process, the input information is transferred from the input layer to the output layer through the hidden layer. If the output layer does not get the desired output, it is transferred to the back propagation. The error signal is returned along the original connection path, and the connection weights between joint in each layer are modified, so the network parameters are adjusted repeatedly to make the minimized error function [16].

BPNN model was performed by using DPS data processing system.

\section{The ARIMA and SARIMA model}

A SARIMA model can be described as ARIMA (p, d, q) multiplied by (P, D, Q), where the terms p, d, q represent ordinary components, while P, D, Q represent seasonal components [17]. Simulated the monthly incidence data of HIV from 2004 to 2017 and the yearly incidence rate of HIV from 1999 to 2016 with the Box-Jenkins modeling approach, we fitted the ARIMA model and the SARIMA model to HIV incidence, and then used the fitted model to out-of-sample predict yearly incidence rate of HIV for the year 2017 and 2018 and monthly incidence of HIV for the year 2018, respectively.

Firstly, autocorrelation function (ACF) graph and partial autocorrelation function (PACF) graph were used to identify the possible values for the autoregressive or moving average components. Secondly, estimates of the model's parameters were obtained by the least squares method according to the different values of $\mathrm{p}, \mathrm{d}, \mathrm{q}$ and $\mathrm{p}$, d, q, P, D, Q. Thirdly, compared the models by the Akaike information criterion (AIC), where the preferred model was the one with the lowest AIC value. Finally the goodness of fit of each model was verified by plotting the autocorrelation and partial autocorrelation of residuals and by using the Ljung-Box test.

SARIMA and ARIMA model was performed by using 11.5 version of Statistical Package for Social Sciences (SPSS11.5) with a significant level of $\mathrm{p}<0.05$.

\section{Performance comparison}

We used ARIMA, BPNN and the seasonal ARIMA model as single time series prediction method to predict yearly and monthly incidence rate of HIV and compare the predictive ability with EMD-BPNN model.

The criterion for comparing the predictive ability of the models was the absolute percentage error defined as:

$$
e=\left|\frac{(x t-\hat{x} t)}{x t}\right| \times 100 \%,
$$

where $x t$ and $\hat{x} t$ denote observed and fitted value at time point respectively. Thus, the preferred model is the one with the lowest mean absolute percentage error.

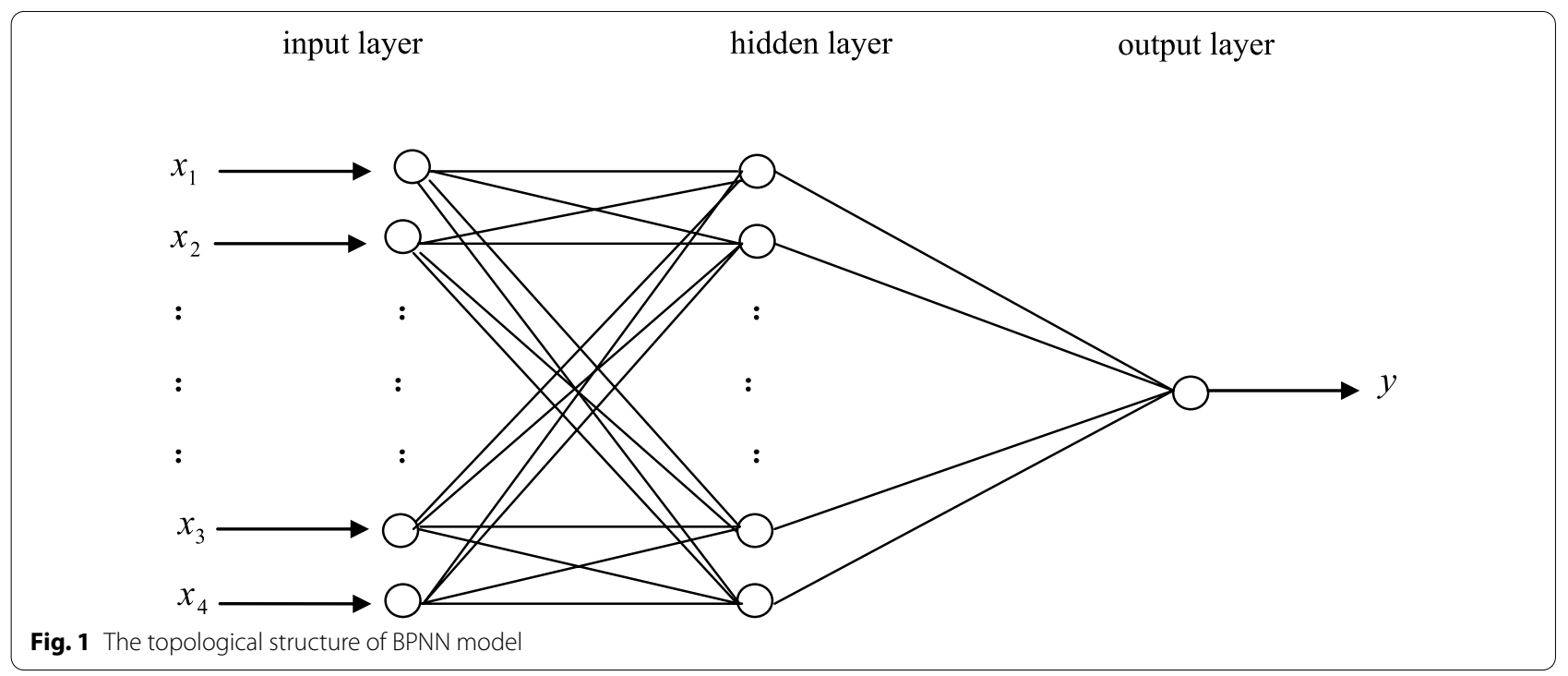




\section{Results}

\section{Descriptive analysis}

From 2004 to 2017, 3310 cases of HIV were reported (3122 males and 188 females) in Dalian, Liaoning Province, China, including 101 fatal cases. The average incidence rate is $4.01 / 100,000$ population, the average mortality rate is $0.12 / 100,000$ population, and the case fatality rate is $3.05 \%$. The incidence of the HIV is mainly concentrated in the age group of 20 to 49 years old which has 2751 cases of HIV reported and coming to $83.11 \%$ in total.

\section{Decomposition results of the original monthly HIV data series by EMD}

The original monthly HIV data series from 2004 to 2017 was decomposed by EMD. Four independent IMFs and one residue item were obtained. Figure 2 showed decomposition results of the original monthly HIV data series of Dalian by EMD. As shown in figure, IMF presents the oscillation characteristics in order from high frequency to low frequency. Specifically, IMF1, IMF2 and IMF3 represented high frequency variable and large fluctuation range, IMF4 represented low frequency component, and the fluctuation range was small. The residue item is the last one, which is reflect the overall trend of the original data series. In this study, the residue item showed that the monthly incidence data of HIV in Dalian during2004 to 2015 is increasing and during 2016 to 2017 is declining.
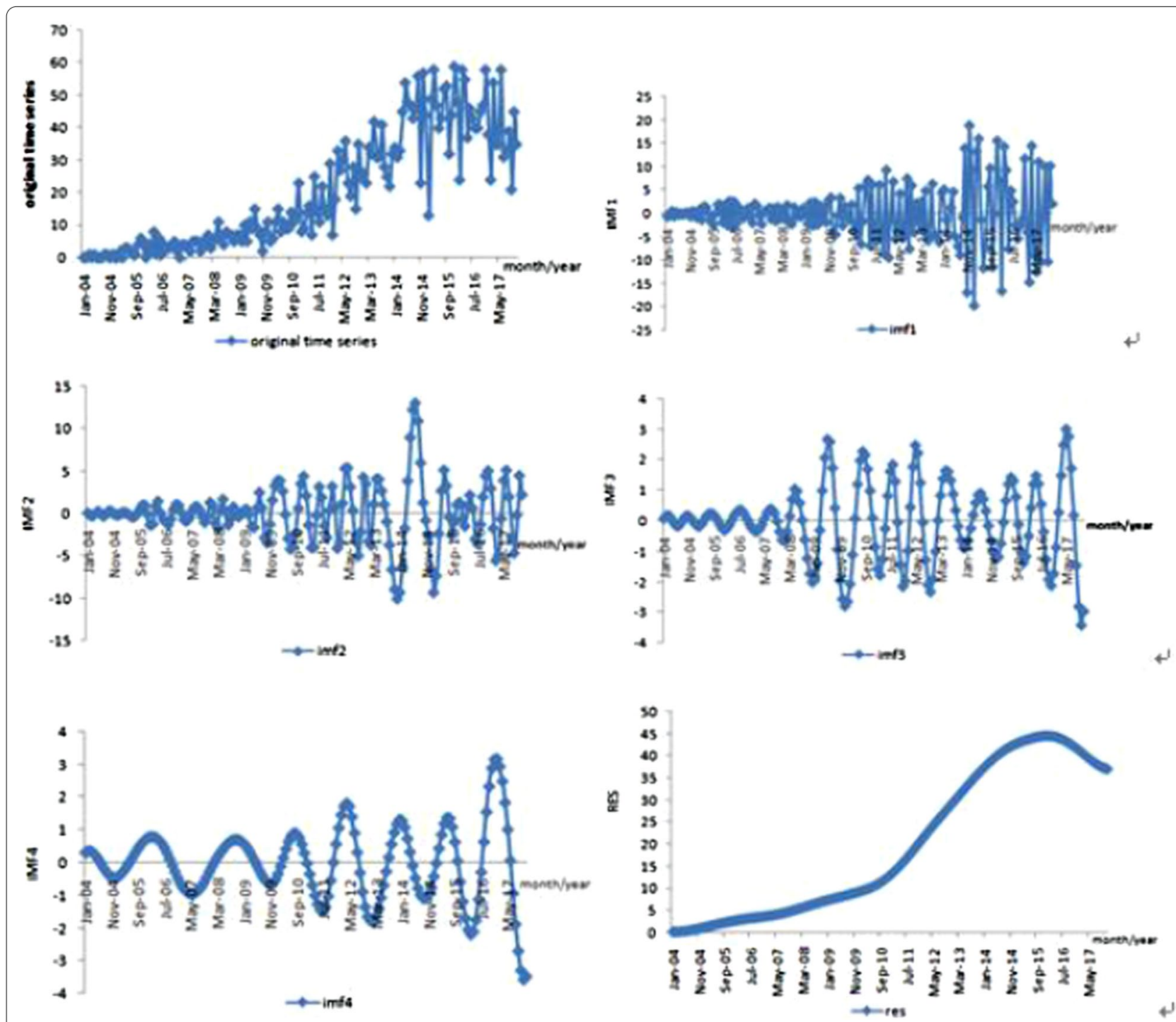

Fig. 2 Decomposition results of the original monthly HIV data series in Dalian by EMD 
Fitting and forecasting IMFs and residue by BPNN model In this study, we establish a BPNN model to predict the decompositions IMF1 to IMF4 and the residue item. Because of the monthly data series, we took the every 12 IMF and residue item as the input, the thirteenth IMF and residue item as output. The parameters of model were as follows. We test 12 candidate the node number of one hidden layer from 1 to 12 , and then, we calculated fitting residuals to determine the optimal value. Table 1 showed the node number of one hidden layer and the model's fitting residuals. The node value was 12 had the smallest fitting residuals $(0.003581 \%)$, so we choice the node number of one hidden layer was 12 , the minimum training velocity was 0.1 , the permissible error was 0.001 , the maximum iteration number was 1000 , and the

Table 1 The different node number of hidden layer and the model's fitting residuals

\begin{tabular}{ll}
\hline The node number of one hidden layer & Fitting residuals (\%) \\
\hline 12 & 0.003581 \\
11 & 0.003717 \\
10 & 0.00381 \\
9 & 0.003985 \\
8 & 0.00363 \\
7 & 0.003737 \\
6 & 0.004291 \\
5 & 0.00458 \\
4 & 0.006355 \\
3 & 0.008446 \\
2 & 0.010625 \\
1 & 0.019218 \\
\hline
\end{tabular}

values of input nodes were standardization transformation. After 1000 run, IMF1 to IMF4 fitting residuals were $0.003581 \%, 0.001022 \%, 0.000354 \%$ and $0.000218 \%$, residue item fitting residuals was $0.00132 \%$, and the model fitted well.

Table 2 showed the number of predicted cases from January to December 2018 obtained from the EMDBPNN model, and the annual incidence equal to the sum of the predicted results of the 12 months. The observed and predicted values in 2018 were relatively close to each other, and the absolute percentage error value for the model was $7.80 \%$.

\section{Performance comparison analysis}

To understand the performance of the hybrid EMDBPNN model, the predicted results of the hybrid EMDBPNN model were compared with BPNN, the seasonal ARIMA and ARIMA.

\section{BPNN}

Because of the monthly data series, we took the every 12 month incidences as the input, the thirteenth month incidence as output. The parameters of model were as follows. The node number of one hidden layer was 12 , the minimum training velocity was 0.1 , the permissible error was 0.001 , the maximum iteration number was 1000 , and the values of input nodes were standardization transformation. After 1000 run, fitting residuals were $0.002840 \%$, and the model fitted well.

Table 3 showed the number of predicted cases from January to December 2018 obtained from the BPNN model, and the annual incidence equal to the sum of the

Table 2 The number of HIV cases observed during 2018 and predicted values obtained from the EMD-BPNN model

\begin{tabular}{|c|c|c|c|c|c|c|c|}
\hline Month & IMF1 & IMF2 & IMF3 & IMF4 & Residue item & Predicted value & Observed value \\
\hline 1 & -0.4262 & -2.6947 & -2.1377 & -3.1996 & 38.7255 & 30.2673 & 28 \\
\hline 2 & -1.2183 & -1.6887 & -1.0954 & -3.1254 & 39.1768 & 32.049 & 30 \\
\hline 3 & 5.6477 & 3.4268 & 0.1188 & -3.193 & 39.5275 & 45.5278 & 46 \\
\hline 4 & -9.2236 & 4.842 & 1.3709 & -3.3332 & 39.9215 & 33.5776 & 50 \\
\hline 5 & 9.1589 & 2.0379 & 2.3587 & -3.4737 & 40.2396 & 50.3214 & 66 \\
\hline 6 & -5.1057 & -1.0484 & 2.5335 & -3.5432 & 40.5752 & 33.4114 & 52 \\
\hline 7 & 0.0473 & -0.8585 & 2.2695 & -3.5595 & 40.9097 & 38.8085 & 45 \\
\hline 8 & 2.6705 & 0.1333 & 1.4116 & -3.5628 & 41.104 & 41.7566 & 41 \\
\hline 9 & 2.1908 & 0.5143 & 0.0681 & -3.5633 & 41.2913 & 40.5012 & 38 \\
\hline 10 & -9.0812 & 0.9662 & -1.0834 & -3.5633 & 41.4653 & 28.7036 & 37 \\
\hline 11 & 7.1544 & 0.6492 & -2.2042 & -3.5633 & 41.5809 & 43.617 & 25 \\
\hline 12 & 1.4365 & -0.7369 & -2.798 & -3.5633 & 41.6835 & 36.0218 & 35 \\
\hline Total & & & & & & 454.563 & 493 \\
\hline $\begin{array}{l}\text { Absolute percent- } \\
\text { age error (\%) }\end{array}$ & & & & & & & 7.797 \\
\hline
\end{tabular}


predicted results of the 12 months. The absolute percentage error for the model was $10.79 \%$.

\section{SARIMA}

Firstly, the sequence of HIV incidence from 2004 to 2017 was used to determine the stability of the sequence. According to Fig. 3, the series show non-stationary mean, so it is necessary to stabilize the variance of HIV incidence by computing its natural logarithm. The monthly data used in this study, so the time series

Table 3 The number of HIV cases observed during 2018 and predicted values obtained from the BPNN model

\begin{tabular}{lll}
\hline Month & Predicted value & Observed value \\
\hline 1 & 42.2638 & 28 \\
2 & 48.471 & 30 \\
3 & 37.5714 & 46 \\
4 & 42.3757 & 50 \\
5 & 56.0465 & 66 \\
6 & 47.1118 & 52 \\
7 & 52.4077 & 45 \\
8 & 43.8192 & 41 \\
9 & 48.7503 & 38 \\
10 & 49.2205 & 37 \\
11 & 30.0177 & 25 \\
12 & 48.1137 & 35 \\
Total & 546.1693 & 493 \\
Absolute percentage & & 10.785 \\
error (\%) & &
\end{tabular}

was differenced once at the seasonal level and non-seasonal level. The transformed HIV incidence showed far less dispersion and become stationary because of that the number of HIV cases fluctuated around the mean. All further statistical procedures were performed on the transformed HIV incidence.

Secondly, because of the time series after one seasonal difference and non-seasonal difference, $\mathrm{d}$ equals one and D equals one. The slow decay in the ACF at lags $1-2$ (autocorrelation $1=-0.578$, autocorrelation $2=0.222$ ) suggests that non-seasonal q equals to one or two, while also it may be zero, and a PACF cutoff at lag 1 (partial autocorrelation $1=-0.578$ ) suggests that non-seasonal $\mathrm{p}$ equals one. The slow decay in the $\mathrm{ACF}$ at lags $11-13$ (autocorrelation $11=0.315$, autocorrelation $12=-0.408$, autocorrelation $13=0.206$ ), and a PACF cutoff at lag 11 (partial autocorrelation $11=0.298$ ) suggests seasonal $Q$ equals zero, one or two and seasonal $\mathrm{P}$ equals zero.

The model parameters for SARIMA $(1,1,2)(0,1,1)_{12}$ model, the non-seasonal autoregressive parameters is estimated as $-0.839(\mathrm{SE}=0.142, \mathrm{t}=-5.925$, $\mathrm{p}=0.000)$, non-seasonal moving average parameters one is estimated as $0.183(\mathrm{SE}=0.176, \mathrm{t}=1.036$, $\mathrm{p}=0.302)$, non-seasonal moving average parameters two is estimated as $0.614(\mathrm{SE}=0.168, \mathrm{t}=3.649$, $\mathrm{p}=0.000)$ and seasonal moving average parameter is $0.952(\mathrm{SE}=0.267, \mathrm{t}=3.567, \mathrm{p}=0.000)$ respectively.

Table 4 showed AIC values for the SARIMA models corresponding to different choices of p, q, P and

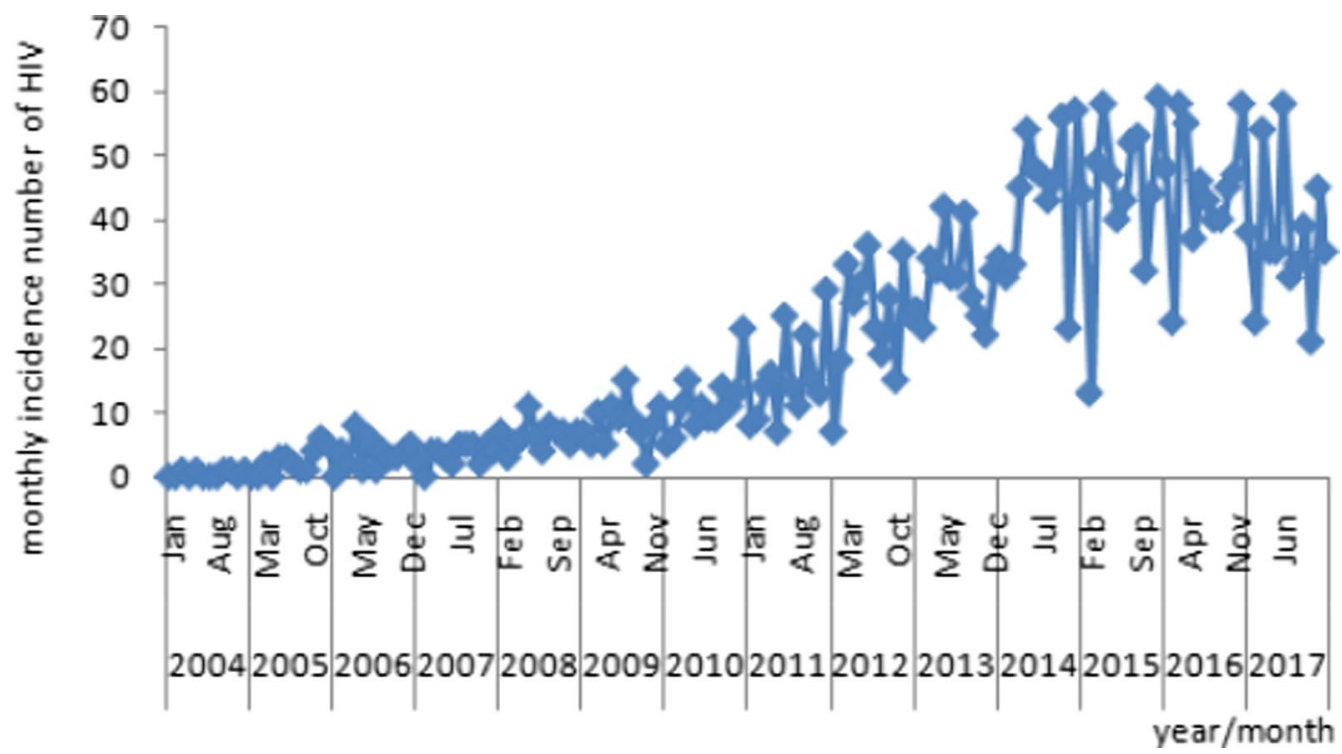

Fig. 3 The sequence of HIV incidence from 2004 to 2017 
Q. The SARIMA $(1,1,2)(0,1,1)_{12}$ model has the lowest AIC.

The Ljung-Box statistic of ARIMA $(1,1,2)(0,1,1) 12$ model showed that there were high $\mathrm{p}$-values associated with the statistics. The null hypothesis of independence in this residual time series cannot be rejected (Table 5). The plots of the ACF and PACF of the residuals show no remaining temporal correlation (Figs. 4, 5). Thus it can be concluded that the ARIMA $(1,1,2)(0,1,1){ }_{12}$ model identified fit the data well.

Table 6 showed out-of-sample predicted values obtained from the SARIMA $(1,1,2)(0,1,1)_{12}$ model and the results were compared with the observed number of HIV cases in 2018. The absolute percentage error value for the model was $9.48 \%$.

\section{ARIMA}

Firstly, the sequence of HIV incidence rate from 1999 to 2016 was used to determine the stability of the sequence. According to Fig. 6, the series show non-stationary mean, so the time series was differenced once at the non-seasonal level. The transformed HIV incidence become stationary because of that the incidence rate of HIV fluctuated around the mean (Fig. 7). All further statistical procedures were performed on the transformed HIV incidence.

Secondly, because of the time series after one non-seasonal difference, d equals one. In the ACF, the coefficients are within the confidence interval (Fig. 7), suggests that non-seasonal q equals zero, and a PACF cutoff at lag 3 (partial autocorrelation1 $=-0.520$ ) suggests that nonseasonal p equals three (Fig. 8).

The model parameters for ARIMA $(3,1,0)$ model, the non-seasonal autoregressive parameters one is estimated as $0.603(\mathrm{SE}=0.196, \mathrm{t}=3.078, \mathrm{p}=0.008)$, autoregressive parameters two is estimated as 0.533 ( $\mathrm{SE}=0.200$, $\mathrm{t}=2.663, \mathrm{p}=0.019$ ), autoregressive parameters three is
Table 5 Autocorrelations analysis results of residuals for SARIMA $(1,1,2)(0,1,1)_{12}$ model

\begin{tabular}{lcllll}
\hline LAG & Autocorrelation & Standard error & \multicolumn{3}{c}{ Box-Ljung Statistic } \\
\cline { 3 - 6 } & & & Value & $\begin{array}{l}\text { Degrees } \\
\text { of } \\
\text { freedom }\end{array}$ & P value \\
& & & \multicolumn{4}{c}{} \\
\hline 1 & -0.043 & 0.081 & 0.276 & 1.000 & 0.599 \\
2 & -0.034 & 0.081 & 0.454 & 2.000 & 0.797 \\
3 & -0.127 & 0.081 & 2.927 & 3.000 & 0.403 \\
4 & 0.002 & 0.080 & 2.927 & 4.000 & 0.570 \\
5 & 0.013 & 0.081 & 2.953 & 5.000 & 0.707 \\
6 & 0.051 & 0.080 & 3.360 & 6.000 & 0.762 \\
7 & -0.099 & 0.080 & 4.902 & 7.000 & 0.672 \\
8 & 0.106 & 0.080 & 6.655 & 8.000 & 0.574 \\
9 & 0.031 & 0.080 & 6.803 & 9.000 & 0.658 \\
10 & -0.052 & 0.080 & 7.236 & 10.000 & 0.703 \\
11 & 0.097 & 0.079 & 8.733 & 11.000 & 0.647 \\
12 & 0.081 & 0.079 & 9.778 & 12.000 & 0.635 \\
13 & -0.013 & 0.079 & 9.805 & 13.000 & 0.710 \\
14 & -0.041 & 0.079 & 10.077 & 14.000 & 0.757 \\
15 & 0.074 & 0.078 & 10.974 & 15.000 & 0.754 \\
16 & -0.062 & 0.078 & 11.608 & 16.000 & 0.771 \\
\hline
\end{tabular}

estimated as $-0.791(\mathrm{SE}=0.204, \mathrm{t}=-3.882, \mathrm{p}=0.002)$ respectively.

The Ljung-Box statistic of ARIMA (3, 1, 0) model showed that there were high $\mathrm{p}$-values associated with the statistics. The null hypothesis of independence in this residual time series can't be rejected (Table 7). The plots of the ACF and PACF of the residuals show no remaining temporal correlation. Thus it can be concluded that the ARIMA $(3,1,0)$ model identified fit the data well.

The predict incidence rates of 2017 and 2018 obtained from the ARIMA $(3,1,0)$ model were 5.569 per 100,000 population and 4.433 per 100,000 population

Table 4 Residual diagnostics for different SARIMA models

\begin{tabular}{|c|c|c|c|c|}
\hline Model & Standard error (SE) & Log-likelihood & $(A I C)$ & $\begin{array}{l}\text { Schwarz } \\
\text { Bayesian } \\
\text { criterion (BIC) }\end{array}$ \\
\hline $\operatorname{ARIMA}(1,1,0)(0,1,0){ }_{12}$ & 0.612 & -133.790 & 271.579 & 277.505 \\
\hline $\operatorname{ARIMA}(1,1,0)(0,1,1){ }_{12}$ & 0.485 & -106.333 & 218.666 & 227.555 \\
\hline $\operatorname{ARIMA}(1,1,0)(0,1,2){ }_{12}$ & 0.462 & -105.583 & 219.166 & 231.017 \\
\hline $\operatorname{ARIMA}(1,1,1)(0,1,0){ }_{12}$ & 0.532 & -113.014 & 232.028 & 240.916 \\
\hline $\operatorname{ARIMA}(1,1,1)(0,1,1){ }_{12}$ & 0.402 & -86.042 & 180.085 & 191.936 \\
\hline $\operatorname{ARIMA}(1,1,1)(0,1,2){ }_{12}$ & 0.424 & -86.563 & 183.125 & 197.939 \\
\hline $\operatorname{ARIMA}(1,1,2)(0,1,0){ }_{12}$ & 0.521 & -109.498 & 226.997 & 238.848 \\
\hline $\operatorname{ARIMA}(1,1,2)(0,1,1){ }_{12}$ & 0.401 & -83.908 & 177.816 & 192.630 \\
\hline $\operatorname{ARIMA}(1,1,2)(0,1,2){ }_{12}$ & 0.403 & -83.842 & 179.685 & 197.462 \\
\hline
\end{tabular}




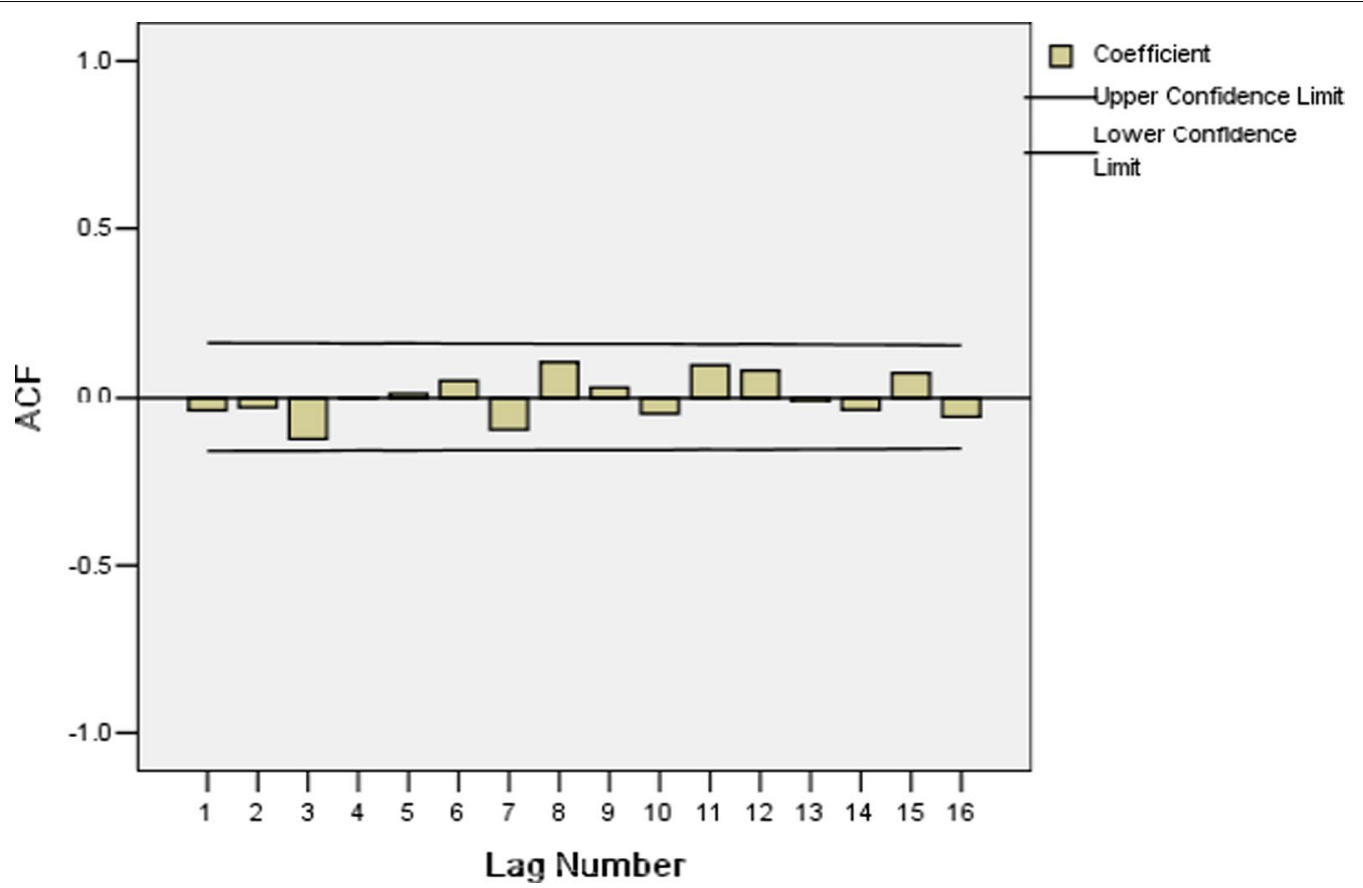

Fig. 4 Autocorrelation function (ACF) of residuals for SARIMA $(1,1,2)(0,1,1)_{12}$ model

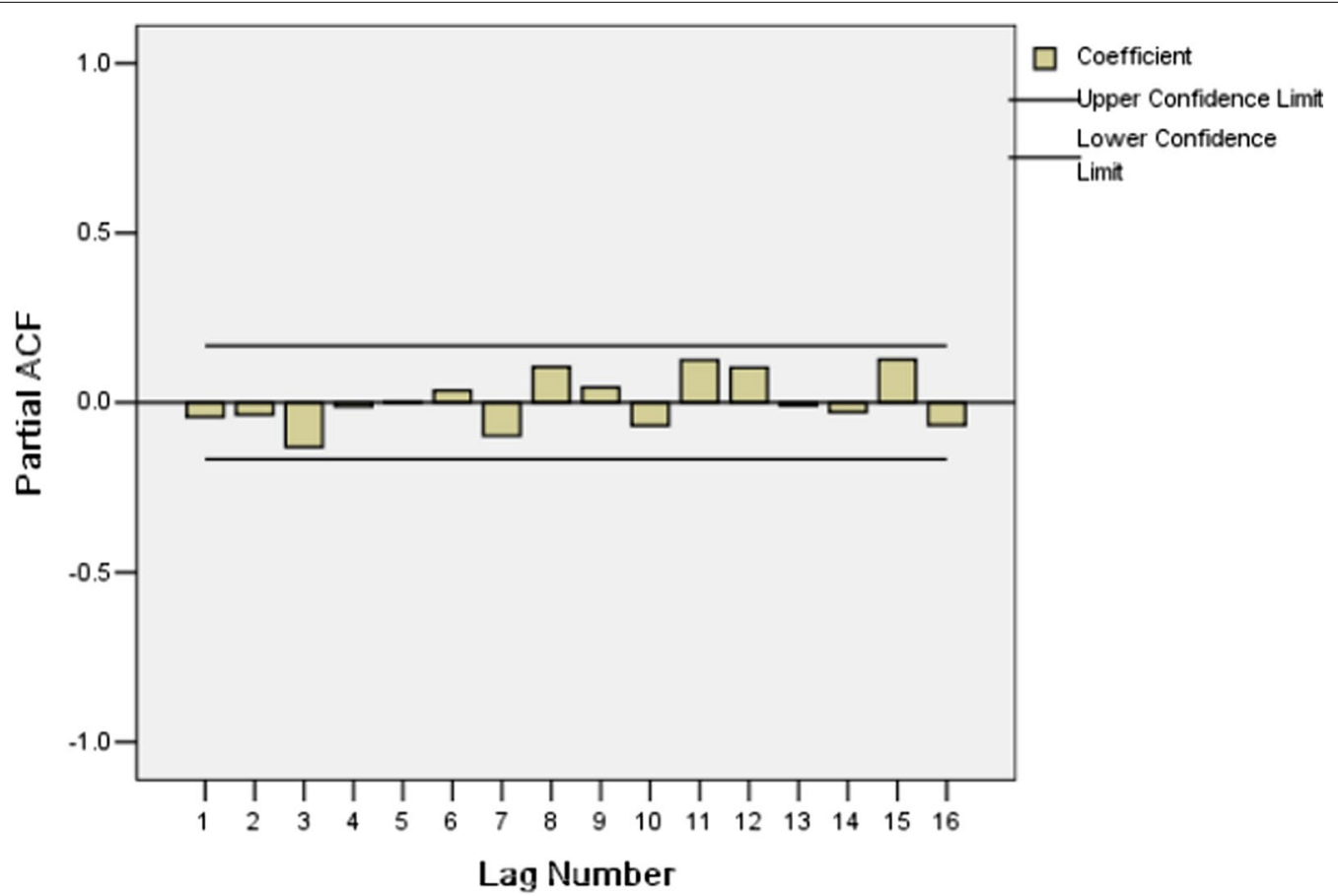

Fig. 5 Partial autocorrelation functions of (PACF) of residuals for SARIMA $\left((1,1,2)(0,1,1)_{12}\right.$ 
Table 6 Observed number of HIV cases in 2018 and predicted values obtained from SARIMA $(1,1,2)(0,1,1)_{12}$ model

\begin{tabular}{lccl}
\hline Month & $\begin{array}{l}\text { Observed } \\
\text { value }\end{array}$ & Predicted value & Predicted value95\%Cl \\
\hline 1 & 28 & 33.128 & $14.607 \sim 75.130$ \\
2 & 30 & 24.133 & $10.639 \sim 54.742$ \\
3 & 46 & 45.609 & $19.774 \sim 105.198$ \\
4 & 50 & 43.043 & $18.563 \sim 99.809$ \\
5 & 66 & 39.427 & $16.782 \sim 92.624$ \\
6 & 52 & 43.186 & $18.297 \sim 101.933$ \\
7 & 45 & 35.672 & $14.906 \sim 85.371$ \\
8 & 41 & 35.021 & $14.572 \sim 84.166$ \\
9 & 38 & 37.461 & $15.431 \sim 90.938$ \\
10 & 37 & 28.312 & $11.602 \sim 69.090$ \\
11 & 25 & 37.745 & $15.270 \sim 93.297$ \\
12 & 35 & 43.519 & $17.519 \sim 108.106$ \\
Total & 493 & 446.256 & \\
Absolute & & 9.482 & \\
percentage & & & \\
error (\%) & & & \\
\hline & & & \\
\hline
\end{tabular}

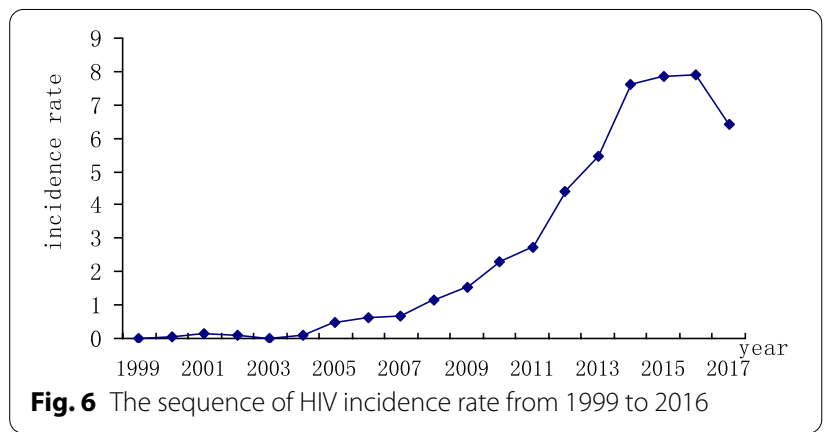

respectively. Compared to the actual value of 5.266 per 100,000 population and 5.041 per 100,000 population, the average absolute percentage error for the model was $8.91 \%$.

\section{Discussion}

As one of the important public health problems in the world, AIDS has caused more and more serious harm to human health. It is one of the three global threats facing human beings, especially in developing countries [18]. According to a new report released by UNAIDS on the eve of World AIDS Day 2021, 1.5 million people were newly infected with HIV in 2020 [19]. The prevention and treatment of AIDS faces great challenges. From 2004 to 2017, 3310 cases of HIV were reported in Dalian, Liaoning Province, China, the average incidence rate is $4.01 / 100,000$ population, the average mortality rate is $0.12 / 100,000$ population, and the case fatality rate is $3.05 \%$. The average incidence rate was lower than national level [20] and higher than the reported average incidence levels of some part of southern city in China $[21,22]$.

The prediction of infectious diseases can detect the trend of disease development in time. Therefore, if an area has continuous HIV surveillance data, the incidence number of HIV can be predicted by establishing mathematical models, and the results could be used to the HIV monitoring and provide the scientific basis for prevention strategies of the area. It makes AIDS prevention and control work more targeted, predictable and initiative. In this study, two stage EMD-BPNN model was established to predict the incidence trend of HIV in Dalian. Firstly, the monthly original incidence data of HIV from 2004 to 2017 was decomposed into a number of component and residue by empirical modal decomposition, then the component and residue items were taken as data sets to establish the neural network model. Two-stage EMDBPNN advantage lied in compared with the prediction model established by direct application of original data, the complexity of the model can be effectively reduced. Because the disease incidence data often had the characteristics of non-linear and non-stationary, but the components obtained through empirical modal decomposition belonged to the variable set with the same frequency, and the residue term belonged to the increasing or decreasing variable, so it was easy to fit.

The other advantage of EMD is that can visually expressed the overall trend of the sequence. As seen from res curve in Fig. 2 during 2004 to 2015 the monthly incidence of HIV is increasing and during 2016 to 2017the monthly incidence of HIV is declining. This may be with the intensification of monitoring and surveillance in the period from 2004 to 2015, the number of newly discovered past infection increased and the number of new HIV-infected persons continued to increase. But with the implementation of preventive and control measures such as national policy, behavioral intervention, health education, et al., the number of new HIV infections declined since 2016. Although the monthly incidence of HIV dropped slightly in 2016 and 2017, but it did not show a significant downward trend. According to the analysis of the past HIV data in Dalian, sexual transmission was the major transmission route and an increase of prevalence was noticed among MSM [23]. So in order to prevent the AIDS epidemic, it is suggested that the health administration department continue to strengthen the efforts and scope for the high risk behavior intervention among MSM.

In this study, to further assess the prediction performance of the hybrid EMD-BPNN model, the absolute percentage error was utilized to measure performance. 


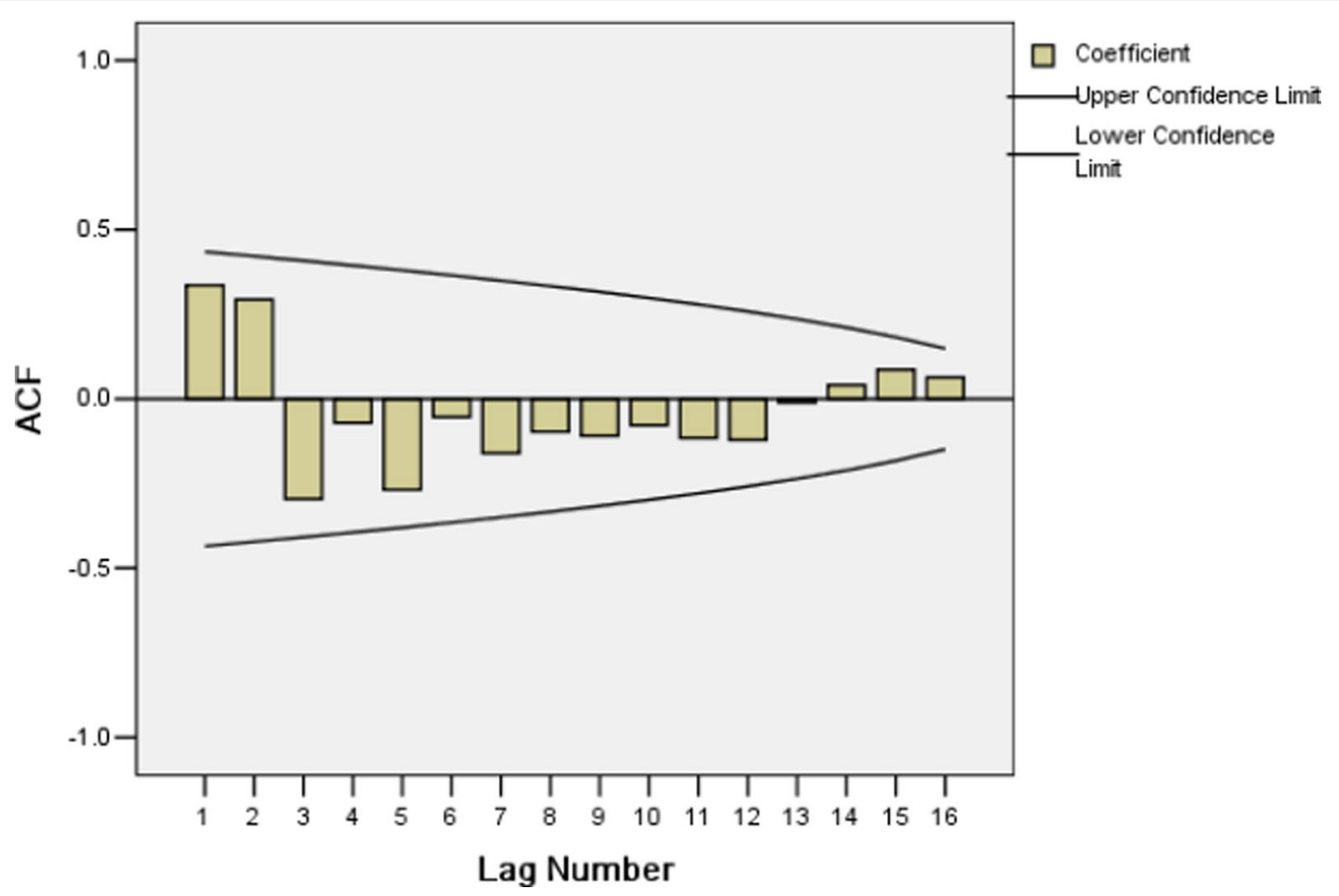

Fig. 7 Autocorrelation function (ACF) for HIV incidence rate series with differenced once at the non-seasonal level

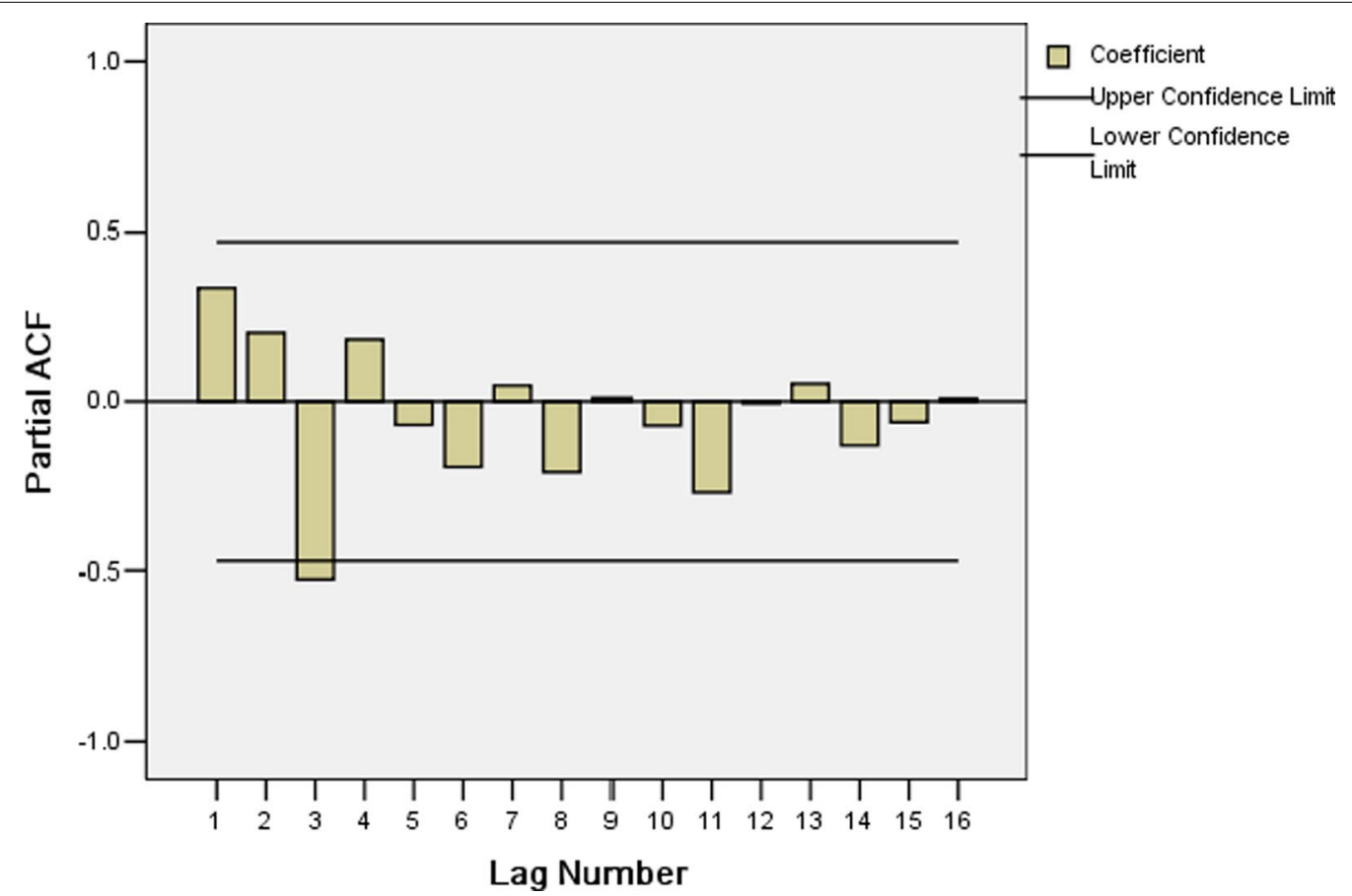

Fig. 8 Partial autocorrelation functions of (PACF) for HIV incidence rate series with differenced once at the non-seasonal level

According to the comparison of EMD-BPNN, BPNN, SARIMA model for monthly HIV data series and ARIMA model for yearly HIV incidence rate series, the SARIMA model performed better than BPNN, but worse than EMD-BPNN model, and the ARIMA model performed slightly worse than EMD-BPNN model. In BPNN model, 
Table 7 Autocorrelations analysis results of residuals for ARIMA $(3,1,0)$ model

\begin{tabular}{lcllll}
\hline LAG & Autocorrelation & Standard error & \multicolumn{3}{c}{ Box-Ljung Statistic } \\
\cline { 4 - 6 } & & & Value & $\begin{array}{c}\text { Degrees } \\
\text { of } \\
\text { freedom }\end{array}$ & P value \\
& & & \multicolumn{4}{c}{} \\
\hline 1 & 0.135 & 0.217 & 0.384 & 1.000 & 0.536 \\
2 & 0.084 & 0.211 & 0.543 & 2.000 & 0.762 \\
3 & -0.199 & 0.204 & 1.489 & 3.000 & 0.685 \\
4 & 0.015 & 0.197 & 1.494 & 4.000 & 0.828 \\
5 & -0.180 & 0.190 & 2.395 & 5.000 & 0.792 \\
6 & -0.041 & 0.183 & 2.446 & 6.000 & 0.875 \\
7 & -0.147 & 0.175 & 3.150 & 7.000 & 0.871 \\
8 & -0.129 & 0.167 & 3.749 & 8.000 & 0.879 \\
9 & 0.030 & 0.158 & 3.785 & 9.000 & 0.925 \\
10 & -0.009 & 0.149 & 3.788 & 10.000 & 0.956 \\
11 & -0.110 & 0.139 & 4.415 & 11.000 & 0.956 \\
12 & -0.114 & 0.129 & 5.193 & 12.000 & 0.951 \\
13 & 0.039 & 0.118 & 5.302 & 13.000 & 0.968 \\
14 & -0.009 & 0.105 & 5.309 & 14.000 & 0.981 \\
15 & 0.067 & 0.091 & 5.848 & 15.000 & 0.982 \\
16 & 0.054 & 0.075 & 6.365 & 16.000 & 0.984 \\
\hline
\end{tabular}

since the calculated data for the infection incidence prediction includes the incidence of infection in the previous period, the prediction effect for the long-term prediction result is poor due to the cumulative error. It is important to point out forecasting model influenced by outbreak for instance COVID-19. According to the results of a study on the impact of COVID-19 on HIV case reporting in China, reports of HIV cases have been significantly affected by COVID-19. Specifically, the number of reported HIV cases has significantly decreased during COVID-19 outbreak [24]. In addition, the proportion of HIV cases reported during the COVID-19 outbreak that were detected late was higher than in the other two nonCOVID-19 outbreak years. In other words, due to the COVID-19, the original pattern of HIV has changed significantly. But when we do infectious disease prediction, we extrapolate it based on the principle of inertia of the data, that is, in the time series the diseases need to be in the same or similar natural state, so if the HIV incidence data of COVID-19 outbreak years and non-outbreak years are mixed together as basic data for prediction, it is bound to occur large errors and even impossible to conduct model. Therefore, in order to understand the trend of HIV in natural state, how to correct the HIV incidence data in the year of COVID-19 outbreak will become an important topic for further study. At the same time, as the incidence of HIV is affected by many natural and social factors, the established prediction model is not immutable. In future research, appropriate prediction model should be re-established according to the Additional file 1 .

This study had several limitations. First, there might be many factors affecting HIV incidence, such as population, economy, behavior and environment, which can all contribute to and interact in the HIV transmission cycle, however, the availability of these data is limited. Second, the data of HIV incidence was obtained from passive surveillance, resulting in a potential underreporting of HIV cases, influencing the precision of our analysis.

\section{Conclusions}

In brief, the original monthly HIV data series were decomposed into four relatively stable IMFs and one residue item using the EMD method, and then all decomposition results are used as training and testing data to establish the BPNN model, the final prediction of monthly HIV data equal to the sum of the predicted results of the each decomposition, and the annual incidence equal to the sum of the predicted results of the 12 months. Compared with BPNN, SARIMA for monthly HIV data series and ARIMA model for yearly HIV incidence rate series, the hybrid EMD-BPNN model has performs better than single times series prediction model like above three models, namely the hybrid model improve the prediction accuracy. Therefore, it can be considered that the hybrid EMD-BPNN model was effective and reliable in predicting the incidence of HIV in Dalian and the results could furnish a scientific reference for policy makers and health agencies.

\section{Abbreviations}

AIDS: Acquired immunodeficiency syndrome; HIV: Human immunodeficiency virus; EMD: Empirical modal decomposition; BPNN: Back-propagation artificial neural network model; IMF: Intrinsic mode function; SARIMA: Seasonal autoregressive integrated moving average; BP-ANN: Back-propagation neural network; ACF: Autocorrelation function; PACF: Partial autocorrelation function; AIC: Akaike information criterion; SPSS11.5: 11.5 version of Statistical Package for Social Science.

\section{Supplementary Information}

The online version contains supplementary material available at https://doi. org/10.1186/s12879-022-07061-7.

Additional file 1: Monthly cases of HIV in Dalian, Liaoning province, China, during 2019 to 2020.

\section{Acknowledgements}

We are grateful to the late the people's Economic Forum users for give us help in writing EMD programs. 


\section{Authors' contributions}

Conceived and designed the study: QA. Collected data: ZZ, JB. Analyzed the data: QA. Wrote the paper: QA. Reviewed the manuscript: JW, JM, XL. All authors have read and approved the manuscript.

\section{Funding}

This research was supported in part by grants from Dalian medical science research program (2011034).

\section{Availability of data and materials}

All data generated or analysed during this study are included in this published article and its additional information files.

\section{Declarations}

Ethics approval and consent to participate

Not applicable.

\section{Consent for publication}

Not applicable.

\section{Competing interests}

The authors declare no conflict of interest.

\section{Author details}

'Dalian Center for Disease Control and Prevention, Dalian 116021, Liaoning, China. ${ }^{2}$ School of Public Health, Dalian Medical University, Dalian 116044, Liaoning, China.

Received: 22 August 2021 Accepted: 15 January 2022

Published online: 29 January 2022

\section{References}

1. Hu BX, Qu B, Liu J, et al. Comparison of ARIMA model and GM $(1,1)$ gray model in forecasting the incidence of AIDS in China. J Prev Med Inf. 2014:30:813-6.

2. Yang YL, Mao SC, Xue $Y$, et al. Prediction on the incidence rate of AIDS in China with $\mathrm{GM}(1,1)$ and trend extrapolation model. Chin J Health Stat. 2014;31:952-4.

3. Wu Z, Rou K, Cui H. The HIV/AIDS epidemic in China: history, current strategies and future challenges. AIDS Educ Prev. 2004;16:7-17.

4. Zhang L, Chow EP, Jing J, et al. HIV prevalence in China: integration of surveillance data and a systematic review. Lancet Infect Dis. 2013;13:955-63.

5. http://www.nhc.gov.cn/jkj/s3586/201312/2b871ccd2ef446eb9542875d3 d68bbca.shtml.

6. Liang $Y X$, Wang $Y F$, Zhai $L$, et al. Analysis of forecasting the incidence of AIDS gonorrhea and syphilis in Jiangsu Province with $\mathrm{GM}(1,1)$ model. J Nantong Univ (Medical Sciences). 2013;1:21-4.

7. Hu BX, Qu B, Liu J, et al. Comparison of ARIMA Model and GM $(1,1)$ Gray Model in Forecasting the Incidence of AIDS in China. J Prev Med Inf. 2014;30(10):813-6.

8. Yang QY, Guo GX, Chen H. Research on AIDS incidence prediction based on ARIMA. China Med Equip. 2016:13:1-4.

9. Wu HL, Qian JS, Xu XD, et al. BP-neural network as a model of predicting STD/AIDS prevalence. Chin J AIDS STD. 2007:6:525-8.

10. Characters of Climatic in Dalian. https://baike.baidu.com/item/\%E5\%A4\% A7\%E8\%BF\%9E/152852?fr=aladdin. Accessed 25 Apr 2019.

11. Li R, TongW ZZJ, et al. Analysis on the surveillance results and epidemiological characteristics of HIV/ AIDS in Dalian. Dis Surveil. 2009;24:852-5.

12. Secretariat of the professional committee on infectious diseases standards and secretariat of the professional committee on parasitic disease standards of the Ministry of Health of the People's Republic of China. Diagnostic criteria of infectious disease. 2010;5.

13. Huang NE, Shen Z, Long SR, et al. The empirical mode decomposition and the Hilbert spectrum for nonlinear and non-stationary time series analysis. Proc R Soc Lond Ser A Math Phys Eng Sci. 1998;454:903-95.

14. Dietmar C, Zhuang XW, Muhammad K, et al. Advances in functional magnetic resonance imaging data analysis methods using Empirical Mode
Decomposition to investigate temporal changes in early Parkinson's disease. Alzheimer's Dementia Transl Res Clin Interven. 2018;4:372-86.

15. Cie Szczyk S, Komada P. Neural network fusion and inversion model for NDIR sensor measurement. 16th Conference on optical fibers and their applications. 2015; 9816:1-7.

16. Tang Q, Feng MG. BP neural network model. In: Tang Q, Feng MG, editors. DPS data processing system experimental design, statistical analysis and data mining. Beijing: Science press; 2007. p. 1056-69.

17. Martinez EZ, Silva EA. Predicting the number of cases of dengue infection in Ribeirão Preto, São Paulo State, Brazil, using a SARIMA model. Cad Saude Publica. 2011;27:1809-18.

18. Yin LJ, Pan LG, Yin XJ. Health education is the effective vaccine for AIDS prevention. Chin J Health Educ. 2000;16:47.

19. Global AIDS Update 2021. https://www.unaids.org/en/resources/docum ents/2021/2021-global-aids-update.

20. Qiao Q, Yang HF. Analysis on the epidemiological characteristics of HIV/ AIDS in China from 2006 to 2015. Chin J Hum Sex. 2019;9:125-7.

21. Ma QJ, Yu B, Ma XC, et al. Discussion on current epidemic status of AIDS in Jiuquan Municipality and prevention measures in low endemic area. Western J Tradit Chin Med. 2014:4:72-5.

22. Wu BJ. Analysis of current situation of HIV/AIDS epidemic in AnXi county. Strait J Prevent Med. 2012;4:38-9.

23. Li R, Tong W, Zhao ZJ, et al. Analysis on the surveillance results and epidemiological characteristics of HIV/ AIDS in Dalian. Dis Surveil. 2009;24(11):852-5.

24. Deng XF, Liu JH, Tang HY, et al. to explore the impact of COVID-19 on HIV/ AIDS case report in Nanning. Chin J Dermatovenereol. 2021;11:1257-61.

\section{Publisher's Note}

Springer Nature remains neutral with regard to jurisdictional claims in published maps and institutional affiliations.

Ready to submit your research? Choose BMC and benefit from

- fast, convenient online submission

- thorough peer review by experienced researchers in your field

- rapid publication on acceptance

- support for research data, including large and complex data types

- gold Open Access which fosters wider collaboration and increased citations

- maximum visibility for your research: over 100M website views per year

At $\mathrm{BMC}$, research is always in progress.

Learn more biomedcentral.com/submissions 